\title{
Modular organization of brain resting state networks in chronic back pain patients
}

\section{Pablo Balenzuela ${ }^{1,2 *}$, Ariel Chernomoretz ${ }^{1,2}$, Daniel Fraiman ${ }^{1,3}$, Ignacio Cifre ${ }^{4}$, Carol Sitges ${ }^{4}$, Pedro Montoya $^{4}$ and Dante R. Chialvo}

1 Consejo Nacional de Investigaciones Científicas y Tecnológicas, Buenos Aires, Argentina

2 Departamento de Física, Facultad de Ciencias Exactas y Naturales, Universidad de Buenos Aires, Buenos Aires, Argentina

${ }^{3}$ Departamento de Matemática y Ciencias, Universidad de San Andrés, Buenos Aires, Argentina

${ }^{4}$ University Institute of Health Sciences Research IUNICS, University of Balearic Islands, Palma, Spain

${ }^{5}$ Department of Physiology, David Geffen School of Medicine, University of California at Los Angeles, Los Angeles, CA, USA

\section{Edited by:}

Jan G. Bjaalie, University of Oslo, Norway

\section{Reviewed by:}

Niels Birbaumer, Institute for Hospitalization and Care Scientific, Italy

Jorge Sepulcre, University of Navarra,

Spain

*Correspondence:

Pablo Balenzuela, Av. Cantilo s/n, Departamento de Física, Pabellón 1, Ciudad Universitaria (1428), Buenos Aires, Argentina.

e-mail: balen@df.uba.ar
Recent work on functional magnetic resonance imaging large-scale brain networks under resting conditions demonstrated its potential to evaluate the integrity of brain function under normal and pathological conditions. A similar approach is used in this work to study a group of chronic back pain patients and healthy controls to determine the impact of long enduring pain over brain dynamics. Correlation networks were constructed from the mutual partial correlations of brain activity's time series selected from ninety regions using a well validated brain parcellation atlas. The study of the resulting networks revealed an organization of up to six communities with similar modularity in both groups, but with important differences in the membership of key communities of frontal and temporal regions. The bulk of these findings were confirmed by a surprisingly naive analysis based on the pairwise correlations of the strongest and weakest correlated healthy regions. Beside confirming the brain effects of long enduring pain, these results provide a framework to study the effect of other chronic conditions over cortical function.

Keywords: functional networks, chronic pain, module organization

\section{INTRODUCTION}

Since the initial work of Eguiluz et al. (2005), complex networks has been widely used to represent functional brain networks. Within this approach, resting state experiments have been analyzed at different scales, either defining nodes as voxels (van den Heuven et al., 2008) or anatomical regions based on a prior automated anatomical labeling (AAL) atlas (Salvador et al., 2005). The organization of resting state brain activity in groups of networks or modules has been studied using different strategies, ranging from univariate analysis as seed correlation (Fox et al., 2005, for example), multivariate linear approaches, as probabilistic independent component analysis (ICA) (Beckmann et al., 2005; Smith et al., 2009) and modular organization in complex networks (Stanberry et al., 2008; Meunier et al., 2009a,b). These analyses has been useful to evaluate the integrity of brain function under normal (Damoiseaux et al., 2006) and pathological conditions (Broyd et al., 2009), including Alzheimer disease (He et al., 2008), schizophrenia (Garrity et al., 2007), and epilepsy (Laufs et al., 2007; Lui et al., 2008), but has not been used in analyzing chronic back pain (CBP) condition.

The experience of pain is usually associated with the activation of a large distributed brain network called the "pain matrix," which includes somatosensory, insular, anterior cingulate and prefrontal cortices, as well as the thalamus (Tracey and Mantyh, 2007). Moreover, previous neuroimaging studies indicate that enduring chronic pain seems to induce a pronounced activation of the pain matrix (Witting et al., 2006) and to alter brain dynamics beyond the feeling of pain itself (Baliki et al., 2008). A recent work (Tagliazucchi et al., 2010) has further reported for the first time alterations in resting state networks of patients with CBP, motivating further work to evaluate possible global changes in their brain modular organization. This is a significant aim, given that patients with chronic pain suffer from a myriad of symptoms including sleep disturbances, depression, cognitive and attention deficits, etc (Apkarian et al., 2004), whose neurophysiological mechanisms are not yet well understood. Thus, in this paper we use fMRI to identify alterations of brain resting state networks in patients with $\mathrm{CBP}$ compared with healthy controls (HC). The modular organization of these networks in both groups is analyzed and significant changes in the communities are contrasted within a pairwise correlations approach.

The paper is organized as follows: Section II describes the experimental conditions. Section II A includes the numerical methods used to construct the functional networks of brain activity and section II B the definition of modularity, node regional roles, and a brief description of the community detection algorithm. Section III describes the community structure of brain functional networks found in both groups together with a detailed analysis of their main features (section III A). Then, a heuristic analysis (section III B) is used to explain the global alterations found in community organization in terms of changes in pairwise correlations. Finally, the identification of the key regions playing a major role in the community organization (section III C) is analyzed. The paper concludes with a discussion of the main alterations found and its consistency with previous findings.

\section{MATERIALS AND METHODS}

Twelve patients with CBP (29-67 years old, mean $\pm \mathrm{SD}=51.2 \pm 11.2)$ and $12 \mathrm{HC}$ subjects $(21-60$ years old, mean $\pm \mathrm{SD}=40.2 \pm 12.7)$ participate in the study. Age differences between both groups are not significant $(p<0.05)$. The study was approved by the Northwestern 
University IRB committee. All participants were right-handed and completed the Depression and the Anxiety Beck's questionnaires, as well as several pain-related data which have been already published elsewhere (Baliki et al., 2006). As already reported in a previous study the depression and anxiety scores in both groups were far away from pathological criteria, and no group differences were observed on these scores. Participants were scanned following a typical brain resting state protocol (Fox and Raichle, 2007), in which the subject is lying in the scanner and asked to keep their mind blank, eyes closed and avoid falling asleep. Functional magnetic resonance data was acquired in all cases using a 3T Siemens Trio whole-body scanner with echo-planar imaging capability using the standard radio-frequency head coil (scanning parameters were similar to those used in an earlier study, Baliki et al., 2008).

In each subject, a total of 300 images were obtained spaced by $2.5 \mathrm{~s}$. in which the brain oxygen level dependent (BOLD) signal is recorded for each one of the $64 \times 64 \times 49$ sites (so-called voxels of dimension $3.4375 \mathrm{~mm} \times 3.4375 \mathrm{~mm} \times 3 \mathrm{~mm}$ ). Preprocessing of BOLD signal was performed using FMRIB Expert Analysis Tool (FEAT, Jezzard et al., 2001), http://www.fmrib.ox.ac.uk/fsl), involving motion correction using MCFLIRT; slice-timing correction using Fourier-space time series phase-shifting; non-brain removal using BET; spatial smoothing using a Gaussian kernel of full-width-halfmaximum $5 \mathrm{~mm}$. Brain images were normalized to standard space with the MNI 152 (average brain image constructed from 152 normal subjects at Montreal Neurological Institute) template using FMRIB's linear image registration tool (FLIRT) ${ }^{1}$. Data was band pass filtered $(0.01-0.1 \mathrm{~Hz})$ using a 0 lag band pass frequency filter in order to avoid low frequency noise (e.g., scanner drift) and high frequency artifacts (Cordes et al., 2000, 2001). An ICA denoising procedure (Beckmann

${ }^{1}$ http://www.fmrib.ox.ac.uk/analysis/research/flirt/. and Smith, 2004) consisting in removal edges and high frequency artifacts by linear regression was done using Melodic (PICA). The data was parceled using anatomical regions based on a prior AAL atlas (Tzourio-Mazoyer et al., 2002). This parcellation divides each cerebral hemisphere into 45 anatomical regions. Regional mean time series were estimated for each individual by averaging the BOLD time series over all voxels in each of the 90 regions, which are used to construct the correlation networks.

\section{A. NETWORKS CONSTRUCTION}

Inherent to the idea behind computing community's properties is the assumption that brain activity can be described as the flow of activity on a dynamic network (Sporns et al., 2004; Salvador et al., 2005; Eguiluz et al., 2005; Achard and Bullmore, 2007; Bullmore and Sporns, 2009; Meunier et al., 2009b). The first step in this direction is to extract the network from the fMRI data. This is shown schematically in Figure 1, where interactions between the ninety regions (Tzourio-Mazoyer et al., 2002) are defined by their mutual co-activity, estimated using partial correlations (Figure 1A). Following (Soranzo et al., 2007), the minimum first order partial correlation between $X$ and $Y$ is calculated as,

$R_{C}(X, Y)=\min _{Z}(R(X, Y \mid Z))$

where

$R(X, Y \mid Z)=\frac{R(X, Y)-R(Y, Z)}{\sqrt{1-R(X, Z)^{2}} \sqrt{1-R(Y, Z)^{2}}}$,

and $R(X, Y)$ is the linear correlation given by

$R(X, Y)=\frac{\langle X(t) Y(t)\rangle-\langle X(t)\rangle\langle Y(t)\rangle}{\sigma(X(t)) \sigma(Y(t))}$,

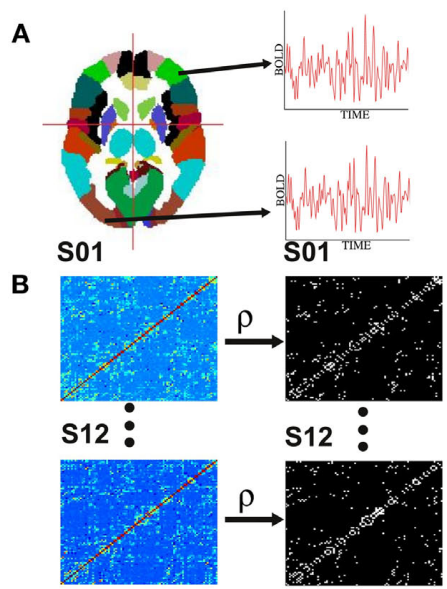

FIGURE 1 | Steps involved in the construction of brain correlation networks. (A) Average BOLD activity time series for each of the 90 anatomical regions defined in (Tzourio-Mazoyer et al., 2002) are extracted. (B) Partial correlation matrices (Eq. 1) for each of the twelve subjects in each group (left) and adjacency matrices (right) with 1 if two regions have a correlation larger
C

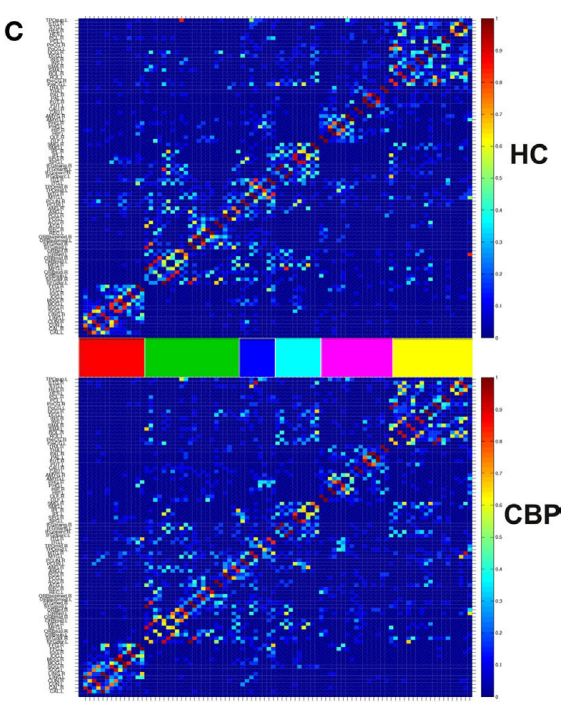

than a certain threshold and 0 otherwise. (C) The weighted group networks obtained from the correlation matrices of the healthy control $(\mathrm{HC})$ (top) and chronic back pain (CBP) (bottom) subjects. The color bars between the panels identify the six communities of the HC group. Same ordering is used for the CBP group. 
where $\langle.$.$\rangle is a temporal average and \sigma(X(t))$ is the standard deviation of $X(t)$. These calculations result in a $90 \times 90$ matrix for each subject (Figure 1B). To define a graph, each matrix is thresholded to create an adjacency matrix $A$ where the $a_{i j}^{\text {th }}$ element is either 1 if the value of the partial correlation is greater than a given threshold $\rho$ or 0 otherwise (Figure 1B).

The threshold level is a critical parameter in this kind of analysis. Very small $\rho$ values will produce highly connected networks in which the presence of well defined communities (i.e., group of nodes which are more densely connected amongst them than with the rest of the network) will be hindered. On the other hand, for very high levels of $\rho$ a disconnected network could appear. Since there is not a priori scale to choose the $\rho$ 's level, we consider a range of threshold values to get networks with a given fixed number of edges, ranging from $N_{L}=100$ up to $N_{L}=400$ links $(\rho \sim 0.6-0.9)$ as was previously done in (Meunier et al., 2009a).

To compare networks between different groups (i.e., healthy subjects or patients) the data can be collapsed into an average correlation matrix for each group (see Meunier et al., 2009a, for example). This results on a single graph for each group, but the information about variability within each group is lost. To overcome this issue, we compute weighted networks calculated as the average of binary networks of all subjects in the group. The corresponding connectivity matrix reads as:

$A_{i, j}=\frac{1}{n_{p}} \sum_{p=1}^{n_{p}} \Theta\left(R_{C}^{p}\left(x_{i}, x_{j}\right)-\rho\right)$

where $n_{p}$ is the number of patients in a given group and $\Theta(X)$ is the Heaviside step function $(\Theta(X)$ is 1 if $X \geq 0$ and 0 if $X<0)$. This means that if partial correlations $R_{\mathrm{C}}$ between regions $i$ and $j$ in a given subject $p$ is above threshold, it contributes with $1 / n_{p}$ to the weight of group matrix between regions $i$ and $j$.

Figure 1C shows the weighted networks estimated from $N_{L}=200$ binary networks for both groups. Regions are sorted according to the community structure found in HC group (see Section 2.2) and their location in the adjacency matrix colored to facilitate their identification in subsequent figures. The differences in network community's organization are described in section III.

\section{B. MODULARITY}

The definition of a network module is usually given in terms of the subset of graph's nodes which are more densely connected among them than with the rest of the network (Newman and Girvan, 2004). Several algorithms have been proposed to detect these modules in weighted complex networks (Clauset et al., 2004; Newman, 2004; Duch and Arenas, 2005). A common approach is to maximize a quantity that estimates the differences between the actual number of intramodule links and the expected number for the same modules in a randomized network. This quantity, called modularity $(Q)$, can be expressed as:

$Q=\frac{1}{2 m} \sum_{i, j}\left[A_{i j}-\frac{k_{i} k_{j}}{2 m}\right] \delta\left(c_{i}, c_{j}\right)$,

where $A_{i, j}$ is the adjacency weighted matrix, $m=1 / 2 \Sigma_{i, j} A_{i, j}$ is the number of links of the network and $k_{i}=\Sigma_{j} A_{i, j}$ is the degree of node $i$ (Newman, 2004; Ahnert et al., 2007) and $c_{i}$ is the community $i$.
To find communities in brain functional networks we use the algorithm developed by Duch and Arenas (2005), which perform an heuristic search procedure based on extremal optimization (Boettcher and Percus, 2001a,b) to find the network community configuration with the best modularity value.

\section{REGION'S ROLES}

In a community-structured network, certain nodes might play more important roles than others in the organization of these structures. A fruitful approach to quantify this contribution is to track the intra-modular degree and the participation coefficient of each node (Guimera and Amaral, 2005). The intra-modular degree is a measure of how well connected is a node compared with other nodes of the same community and its $z$-score can be computed as,

$z_{i}=\frac{\kappa_{i}-\bar{\kappa}_{c_{i}}}{\sigma_{\kappa_{c_{i}}}}$,

with $\kappa_{i}$ the intra-modular degree of the $i$ th node, i.e., the number of links of node $i$ to other nodes in its module $c_{i}, \bar{\kappa}_{c_{i}}$ as the average of $\kappa_{i}$ over all nodes in module $c_{i}$ and $\sigma_{\kappa_{c_{i}}}$ the standard deviation of $\kappa$ in $c_{i}$.

The participation coefficient quantifies the balance between the intra-module versus inter-module connectivity for a given node. It is defined as

$P_{i}=1-\sum_{s=1}^{N_{M}}\left(\frac{\kappa_{i c}}{k_{i}}\right)^{2}$,

where $\kappa_{i c}$ is the number of links of node $i$ to nodes in module $c$ and $k_{i}$ is the degree of node $i$. If all the links of node $i$ fall within its own module, then $\kappa_{i c}=k_{i}$ and the participation coefficient is 0 . On the other hand, if all the links of node $i$ are distributed among all other modules, $P_{i}$ approaches one.

Considering these two definitions, nodes with high intra-modular degree are considered hubs, while nodes with high participation coefficients are called connectors. These definitions are used here to identify the regions which play key roles in the organization of brain activity at resting state.

\section{RESULTS}

In this section we will show the results related with the analysis of the structure of functional brain networks. First, we will describe the communities found in both groups, their stability properties and their functional implications. Second, we will analyze which changes in pairwise correlation explains the alterations seen in the $\mathrm{CBP}$ communities, and finally we will identify the regions which play key roles in module's organization.

\section{A. COMMUNITIES}

The algorithm used to search for partitions which maximizes the network modularity is shown schematically in Figure 2A. For the two groups studied as well as for an equivalent random networks, the maximum modularity values are shown in Figure 2B as a function of $N_{L}=\left(N_{L} 100-400\right)$. Notice that both networks show values of $Q_{\max }$ larger than their equivalent random networks, implying a highly modular structure. Although $Q_{\max }$ depends on $N_{L}$, no significant differences where found between HCP and CBP, for any 
A

Weighted Network Maximizing $\mathbf{Q}$

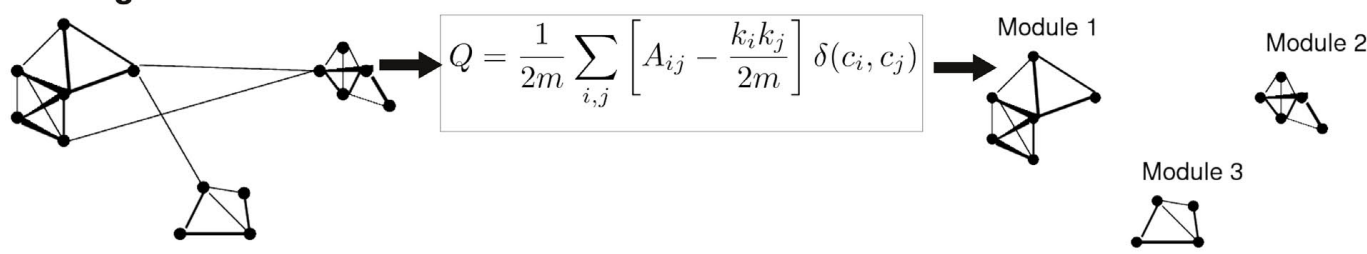

B

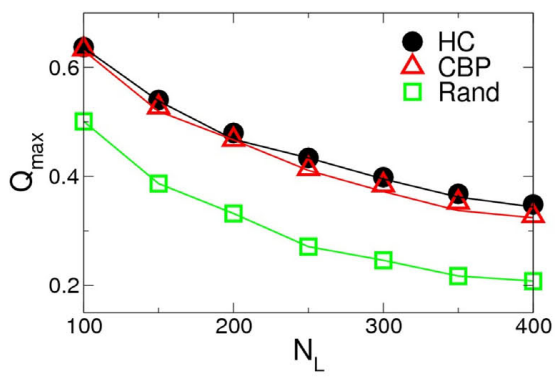

D

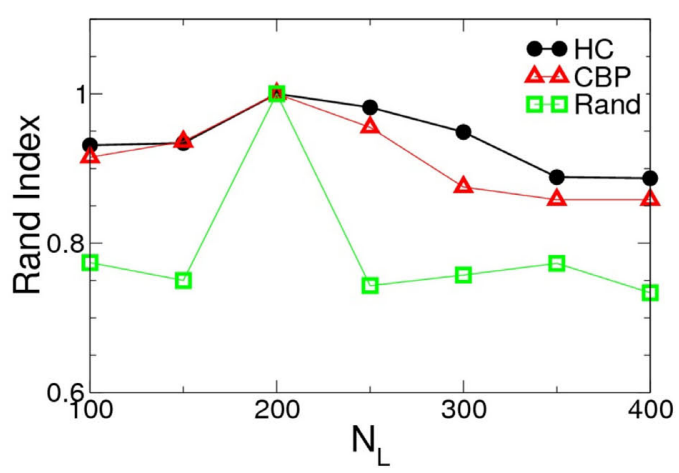

FIGURE 2 | (A) Schematic representation of the algorithm to find modularity (Eq. 5) identifying nodes mutually connected more strongly than with the rest of the network. (B) Changes in the maximum modularity as a function of the number of links, $N$ for both groups as well as for an equivalent random network. (C) Number of modules at $Q_{\text {max }}$ as a function of $N_{L}$ for both groups. (D) Robustness of the communities structures. Rand index of partitions obtained for different values of $N_{L}=\{100,150,250,350,400\}$ compared with those of $N_{L}=200$
C

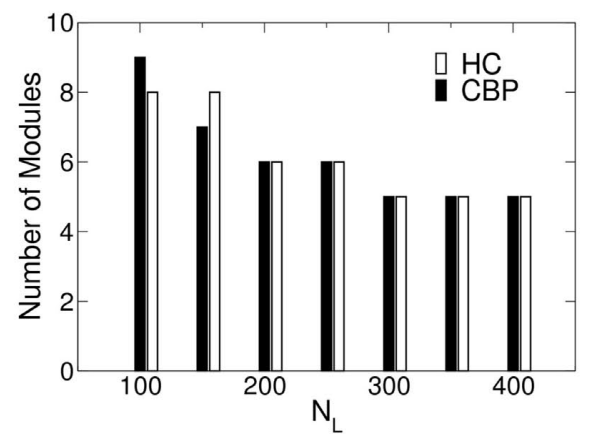

E

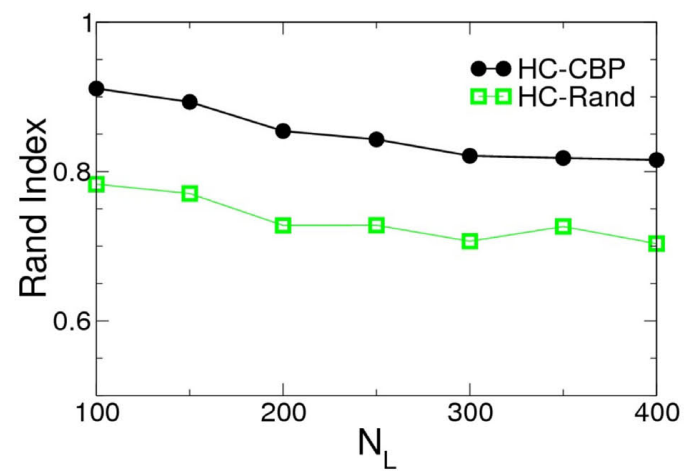

for $\mathrm{HC}$ (black circles), CBP (red triangles), and equivalent random weighted networks (green squares). (E) Rand index between partitions of $\mathrm{HC}$ and $\mathrm{CBP}$ (black circles) as a measure of module's similarity in both groups. The Rand index is also calculated between $\mathrm{HC}$ and equivalent random weighted networks (green squares). The difference between both groups community' structures is smaller than the difference expected between $\mathrm{HC}$ and an equivalent random network. given $N_{L}$. The number of detected modules as a function of $N_{L}$ is shown in Figure 2C. The number of communities stabilizes for $N_{L} \geq 200$ and is similar for both groups.

To further investigate possible differences between groups, the sets of modules found at $Q_{\max }$ were compared, using the Rand index (RI) (Rand, 1971). The RI is a coefficient that quantifies the percentage of agreement between two partitions ( $X$ and $Y$ ) of a given set of elements. Thus, $\mathrm{RI}=1$ if both partitions $X$ and $Y$ are exactly the same (i.e., they have the same quantity of modules and the same integrants en each one) and RI $=0$ if they are completely different.

In Figure 2D we shown the calculated RI between networks of different $N_{L}$ with the one corresponding to $N_{L}=200$ for HC, CBP, and a random realization. No major changes in community organization 
for brain networks is observed as a function of $N_{L}$, but large changes are shown in equivalent random networks. This result suggest that the observed structures are a robust feature of the brain networks.

Figure 2E shows the RI between HC and CBP partitions (black circles) as a function of $N_{L}$, with the RI calculated between $\mathrm{HC}$ and a random equivalent network (green diamond). The differences found between HC and CBP are much smaller than those expected between $\mathrm{HC}$ and equivalent random networks. These results suggest that more detailed analysis is needed to identify potential differences between control subjects and patients. In order to do this we will restrict further analysis to networks with a fixed $N_{L}$. Since the number of communities at $Q_{\max }$ stabilizes for $N_{L}=200$, such value was chosen as representative.

The modular structure in which the brain network organizes (for $N_{L}=200$ ) are shown in Figure 3 depicted in two ways. In the left panels we use an anatomical representation with the coordinates of
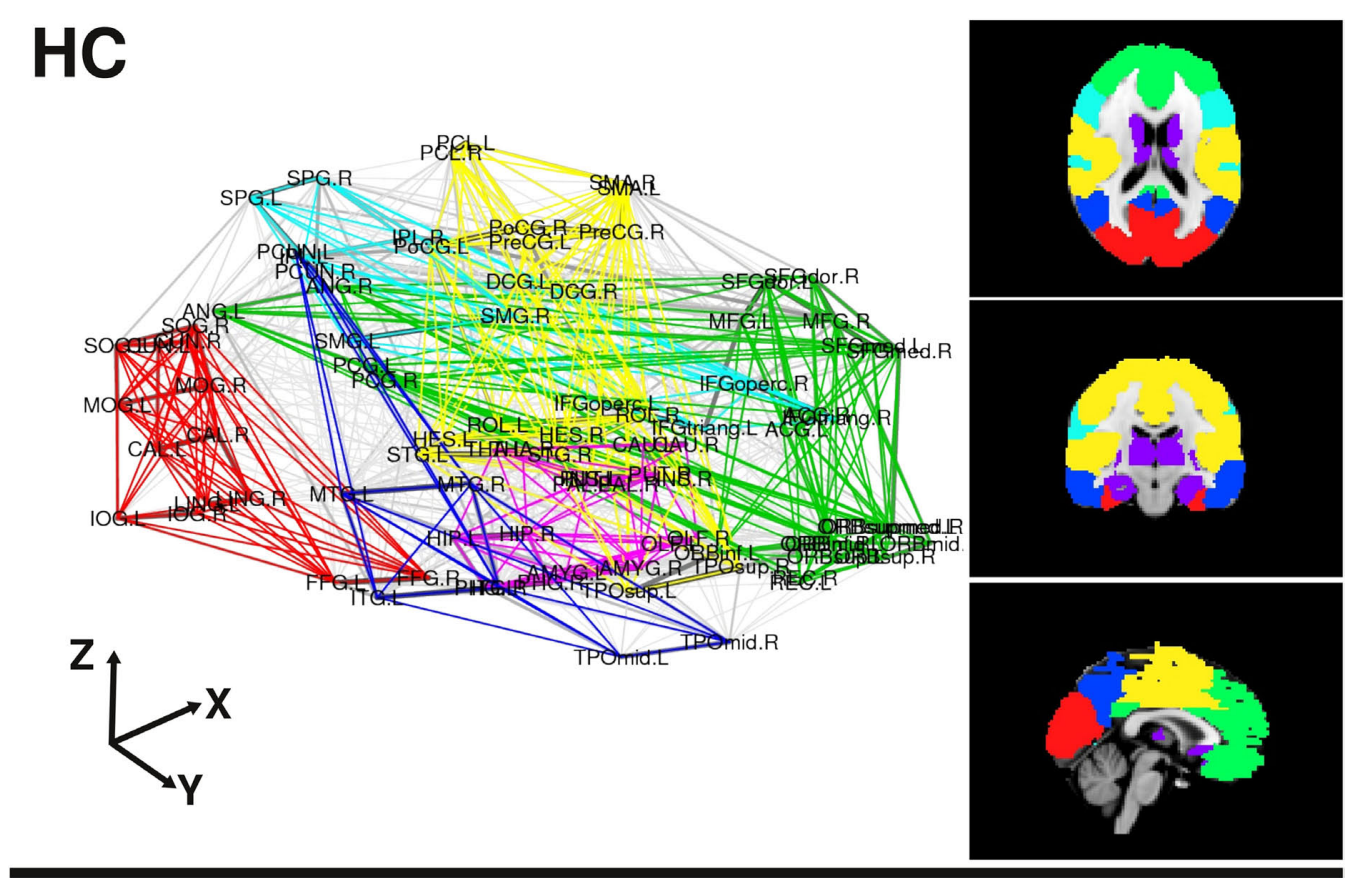

\section{CBP}
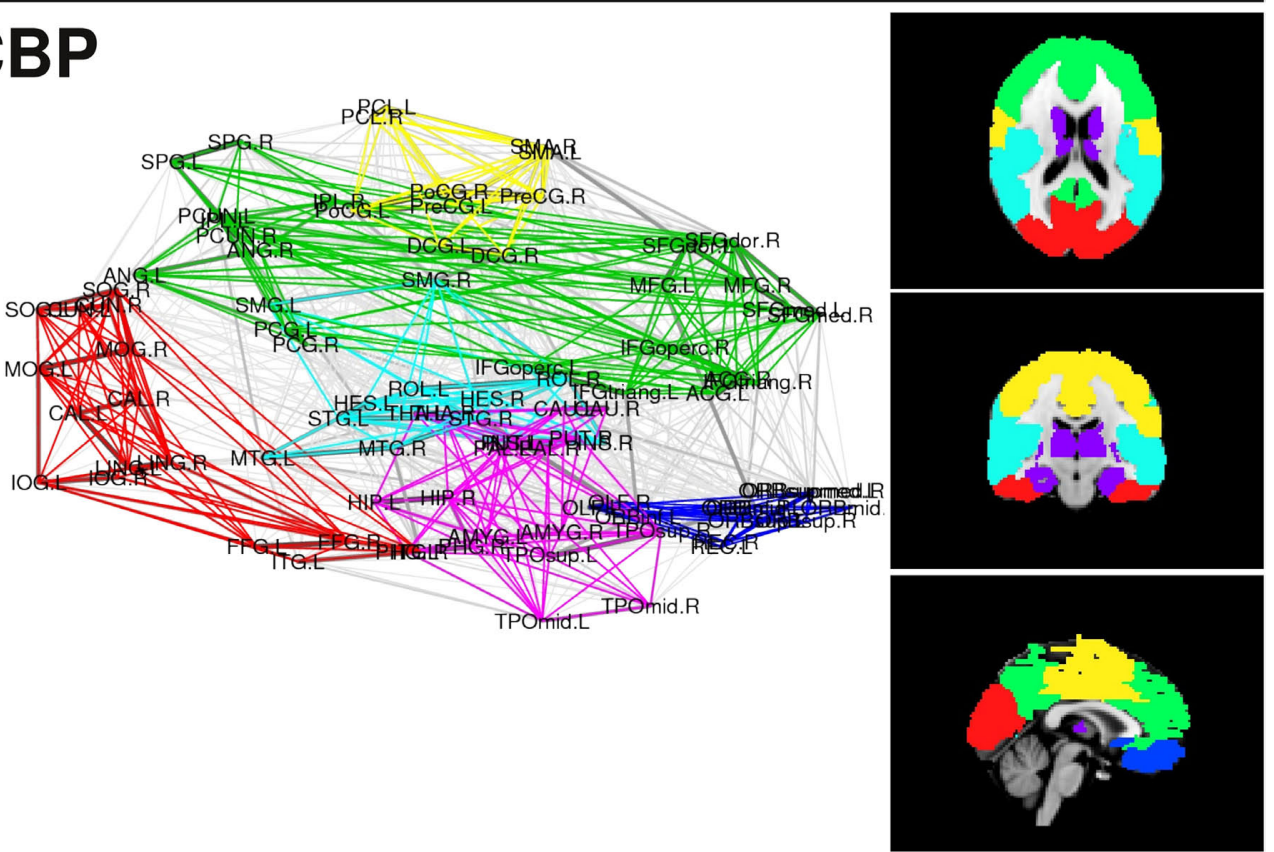

FIGURE 3 | Community organization for both group networks in two representations. Left panels use the coordinates of the center of mass of each region, the right panels depict axial, coronal and sagittal views of the found communities over a MNI-152 template atlas. Modules are colored as listed in Table A2 (see Appendix). 
the center of mass of each region. Right panels display a brain representation in axial, coronal, and sagittal views of found communities over a MNI-152 template atlas. We can observe that both, the HC and the CBP group networks, show a structure formed by up to six communities which are listed in Table A2, along with the membership for each community (see Appendix). Abbreviations used for brain regions (as in Salvador et al., 2005) can be found in Table A1 (see Appendix).

A detailed analysis of these findings can be summarized as follows (see also Figure A1 in Appendix): The first community (red/ visual) involves several regions of the lateral and medial visual pathways (calcarine, cuneus, lingual, and occipital gyrus) and could be related to the visual resting state network postulated by Beckmann et al. (2005). This community appears to extend to the inferior temporal gyrus in CBP, but not in HC. A second community (green/ fronto-medial) was mainly composed of several frontal regions (superior and inferior frontal gyrus, orbito-frontal cortex), as well as anterior cingulate and angular gyri. This community could be related to two resting state networks: the default mode (DMN) and the executive control networks (Beckmann et al., 2005). This community appears to be altered in CBP patients, because it extends to the precuneus and the medial frontal cortex in CBP patients, but does not include the orbito-frontal cortex as it occurs in HC. The third community (blue/parieto-temporal) is formed by the precuneus and the temporal lobes in $\mathrm{HC}$, displaying similarities to Beckmann's DMN. This community extends to the orbito-frontal cortex in CBP patients, but not in HC. The fourth community (light blue/fronto-parietal) involves regions from the inferior frontal cortex, the superior parietal and the supramarginal gyrus, and could be associated with two resting state networks: the auditory system and the dorsal visual stream (Beckmann et al., 2005). This community extends to the insula in CBP patients, but not in HC.
The fifth community (magenta/subcortical) is comprised by subcortical regions, such as hippocampus, thalamus, basal ganglia, and amygdala. No differences between groups appeared in the module membership of this community. The last community (yellow/ sensorimotor) includes the sensorimotor cortices as well as other regions of the pain network such as cingulate gyrus and insula (Zaki et al., 2007). This community appears to be altered in CBP patients because it does not include the insula and the posterior portion of the cingulate gyrus as it occurs in HC. A side by side comparison between $\mathrm{HC}$ and $\mathrm{CBP}$ communities and Beckmann's resting state networks can be found in Figure A1 of Appendix.

\section{B. PAIRWISE CORRELATIONS SUFFICE TO EXPLAIN COMMUNITIES CHANGES?}

The results displayed in Figure 3 show that the observed changes in modular organization are basically of three types: (I) there are communities that split into two or more subgroups, (II) there are modules that fuse together forming a single community, and (III) nodes are adsorbed into other communities, and some communities disappear. Admitting that these changes can be brought about by a complex combination of factors, we test here for the simplest of all cases, which is that linear bivariate correlation changes can be sufficient to explain any of the three types of communities changes.

Thus we re-analyze the interactions between nodes through pairwise correlations. An important point is that we start taking the community partition of the HC (Figure 3 top) as a reference frame and compute the mean intra-modular correlation, $\bar{r}_{\mathrm{IM}}$, for each of the communities defined in this reference $\mathrm{HC}$ group. The idea is that $\bar{r}_{\mathrm{IM}}$ should estimate the strength of the bonds holding together the communities, and that changes should occurs preferentially in weakly coupled modules. Figure 4A shows $\bar{r}_{\mathrm{IM}}$ for each
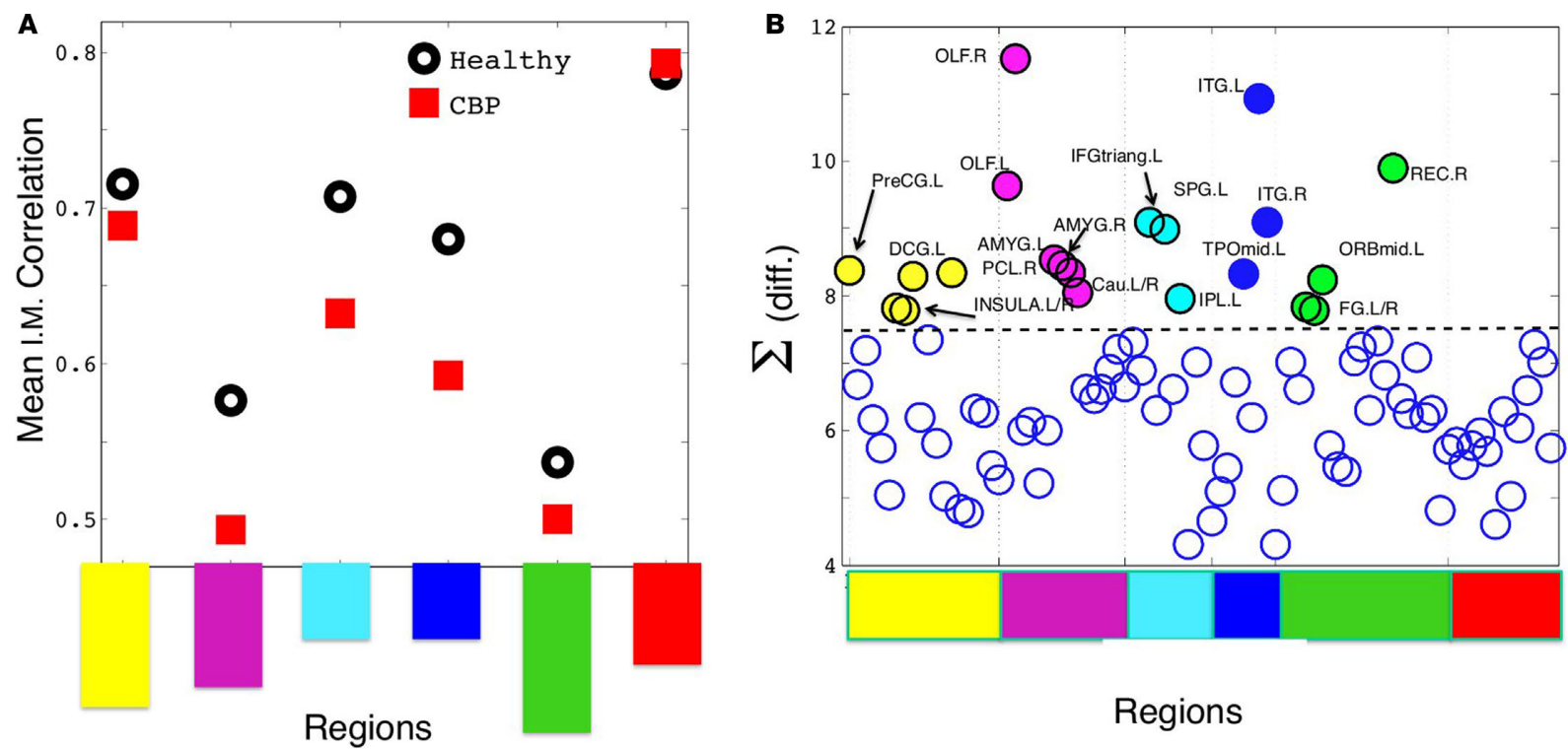

Regions

FIGURE 4 | (A) Mean intra-modular correlation for both groups. Notice that, with one exception, all CBP modules show weaker correlations than the corresponding HC communities. (B) The summatory of the HC-CBP absolute differences in pairwise correlation computed for each brain region. Regions with the biggest changes (labeled), hold the key to understand the community changes. Communities labeled with the same colors as in Figures 1C and 3;Table. 
of the six communities in both groups. It can be seen that CBP patients exhibit a mean intra-modular correlation smaller that control subjects, with the exception of the Posterior module (red). This is consistent with the expectation that communities with weakest correlations should be the ones more easily segregated by potential pathological changes in the interactions.

Thus we focus at the weight (interaction) changes within each community. The largest changes respect to the $\mathrm{HC}$ hold the key to understand all the community alterations. We start by computing the summatory of correlations for each node (plotted in Figure 4B) and inspecting the nodes exhibiting the largest (absolute) change in its correlations. Then, we look whether these changes can explain directly any of the structural changes observed in a given community.

The corresponding observed changes, as listed below (see Figure 5):

(a) The parieto-temporal community (Coded blue in HC, Figure 5A) is not present in CBP. In this module all the pair correlations have decreased (range $[-0.2,-0.1]$ ) in CBP, breaking this structure apart. Larger correlation levels observed in CBP than in HC between inferior temporal gyrus (ITG) and lingual (LING), fusiform (FFG), and inferior occipital gyrus (IOG) $(+0.15)$ result in the former (ITG) being included in the posterior community (red).

(b) The fronto-medial (green) community observed in $\mathrm{HC}$ splits in two subgroups: a new CBP (orbito-frontal, blue) community is created and the fronto-medial CBP community (green) is reorganized, (see Figure 5B). These changes can be explain by the increased interaction between olfactory (OLF), the orbital part of the superior frontal gyrus (ORBsup) and the gyrus rectum (REC) $(+0.16)$ in CBP. Simultaneously the correlation between the orbital part of the middle frontal gyrus (ORBmid) and angular (ANG), middle frontal (MFG), and anterior cingulate gyrus (ACG) decreased $(-0.12)$. This two opposite behaviors give raise to a mean intra-modular correlation that do not change considerably in CBP.

(c) The same type of behavior can be observed for the Sensorimotor $\mathrm{HC}$ community (yellow, Figure 5C). In this case, the interaction between the insula and the precentral gyrus (PreCG), the paracentral lobule (PCL), and superior temporal gyrus of temporal pole (TPOsup) are lower in CBP than in $\mathrm{HC}(-0.13)$. In addition, the interactions between rolandic operculum, supplementary motor area (SMA) and precentral gyrus (PreCG) are decreased $(-0.12)$, whether correlations between insula and Heschl gyrus in CBP are increased $(+0.11)$.

(d) The fronto-parietal (light-blue) community observed in HC is adsorbed by the fronto-medial module in CBP (Figure 5D), mainly because the interaction between superior parietal (SPG) and supramarginal (SMG) has increased $(+0.15)$.

(e) In addition, the supramarginal (SMG) region is left apart from the fronto-parietal HC module in CBP (Figure 5E). This happens because the interaction between this and inferior parietal (IPL), the opercular part of the middle frontal gyrus (IFGoperc) and the triangular part of the inferior frontal gyrus (IFGtriang) is decreased $(-0.14)$. In this case all the regions involved in the new community have increased their internal interactions (superior temporal, supramarginal and middle temporal gyrus, insula, and Heschl operculum).

The mentioned arguments show that the changes observed in the CBP networks can be explained by looking at the pairwise interaction changes within each community and focusing on the largest correlation changes respect to the HC. At some point this is to be expected as the algorithm uses precisely these correlations when it assign each node to a given community.

\section{HUBS AND CONNECTORS}

The results discussed in the previous section show that both CBP and HC networks are structured in the same number of communities, although they show notorious differences in organization and region's membership. This produces that, within these communities, certain key regions present different connectivity. To further quantify these differences, the $z$-score of the intra-modular degree $\left(z_{i}\right)$ and the participation coefficient $\left(P_{i}\right)$ of each node were computed (e.g., Eqs. 6 and 7). This technique allows the labeling in each case of hubs and connectors for each module as it is presented in the top panels of Figure 6 (see names for each region further detailed in Table 1).

The location of hubs and connectors provide clues about the key players in each module organization, thus it is important to study the changes in role of these nodes between the groups. This is plotted in the bottom panels of Figure 6 for the intra-modular degree and participation coefficient of Healthy Controls $z_{i,(\mathrm{HC})}$ and $P_{i,(\mathrm{HC})}$ versus Chronic Back Pain patients, $z_{i,(\mathrm{CBP})}$ and $P_{i,(\mathrm{CBP})}$. If there were no changes in $z_{i}$ and $P_{i}$, all points should collapse in the diagonal, thus, distance from the diagonal can be interpreted as a measure of region's role change. In the plot, those regions whose change in $z_{i}$ or $P_{i}$ are more than two standard deviations are highlighted. Concerning hubs, note that inferior temporal gyrus (ITG.L and ITG.R), superior frontal gyrus, medial orbital (ORBSupMed.R), middle temporal gyrus (MTG.R) and superior frontal gyrus, medial (SFGmed.R) are regions with high intra-modular degree in $\mathrm{HC}$ and low intra-modular degree in CBP patients. Superior frontal gyrus, orbital part (OrbSup.L) is a region with high intra-modular degree in CBP and low in HC. Concerning connectors, caudate (CAU.R), superior frontal gyrus, medial orbital (ORBSupMed.R), superior frontal gyrus, medial (SFGmed.R) and anterior cingulate, and paracingulate gyri (ACG.L) are regions whose participation coefficient increase notoriously in CBP respect to HC groups.

For any given node, changes in its $z_{i}$ or $P_{i}$ value could arise basically due to two mechanisms: local changes, i.e., when there are changes in its own connectivities (by increasing or decreasing the number of links with the rest of the network) or global changes, i.e., due to changes in the mean intra-modular degree by alterations in other connections or by a change in module memberships. These is demonstrated by the results in Table 2 which shows the degree of these regions and compare with $\left\langle k_{i}>\right.$ of regions who belong to.

\section{DISCUSSION}

The major aim of the present study was to examine the modular organization of functional brain networks in CBP. To characterize the modularity of these complex networks, we applied tools from 


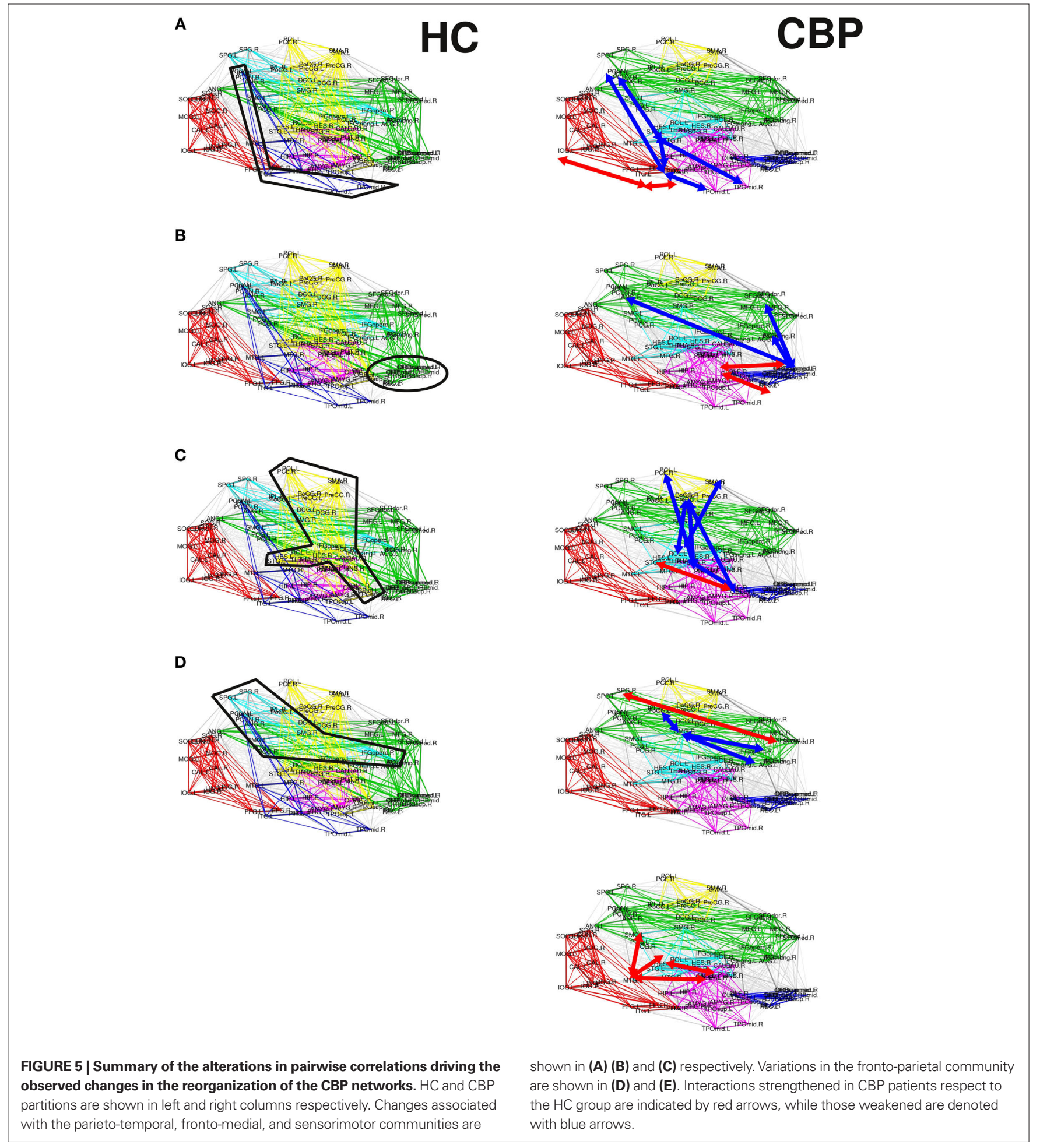

graph theory to BOLD time series obtained from resting fMRI. The present findings were derived from purely data driven analyses and confirmed by a heuristic approach using pairwise correlations. Based on previous evidence showing extensive alterations of brain dynamics in chronic pain patients (Baliki et al., 2006, 2008; Geha et al., 2008; Tagliazucchi et al., 2010), we hypothesized that topological properties of these functional brain networks (i.e., number and components of the modules, intra-modular degree, participation coefficient of the nodes) should be altered as a consequence of persistent pain. Our results revealed significant group differences in the modular organization of several brain resting state networks, rather than global changes of brain modularity. Basically, we observed that $\mathrm{HC}$ and $\mathrm{CBP}$ patients differed in the connectivity of frontal lobes and anterior cingulate gyrus, which are usually involved in the default mode resting 
Table 1 | Regions identified as hubs or connectors for each of the communities in both groups.

\begin{tabular}{|c|c|c|}
\hline Module & Hub & Connector \\
\hline \multicolumn{3}{|l|}{ HC } \\
\hline Visual & Superior occipital gyrus (SOG.R) & Fusiform gyrus (FFG.L) \\
\hline Fronto-medial & Superior frontal gyrus, medial (SFGmed.R) & Inferior frontal gyrus, orbital part (ORBinf.L) \\
\hline Parieto-temporal & Middle temporal gyrus (MTG.R) & Precuneus (PCUN.L) \\
\hline Fronto-parietal & Inferior parietal (IPL.L) & Superior parietal (SPG.L) \\
\hline Subcortical & Hippocampus (HIP.R) & Caudate nucleus (CAU.L) \\
\hline Sensorimotor & Supplementary motor area (SMA.R) & Superior temporal gyrus (TPOsup.L) \\
\hline \multicolumn{3}{|l|}{ CBP } \\
\hline Visual & Fusiform gyrus (FFG.R) & Inferior temporal gyrus (ITG.L) \\
\hline Fronto-medial & Superior frontal gyrus, dorsolateral (SFGdor.L) & Superior parietal gyrus (SPG.R) \\
\hline Orbito-frontal & Superior frontal gyrus, orbital part (ORBsup.L) & Inferior frontal gyrus, orbital part (ORBinf.L) \\
\hline Fronto-parietal & Superior temporal gyrus (STG.L) & Middle temporal gyrus (MTG.R) \\
\hline Subcortical & Hippocampus (HIP.R) & superior temporal gyrus (TPOsup.R) \\
\hline Sensorimotor & Supplementary motor area (SMA.R) & Median cingulate and paracingulate gyri (DCG.R) \\
\hline
\end{tabular}

Table 2 | Changes in node's role.

\begin{tabular}{|c|c|c|c|c|c|c|c|}
\hline \multirow[t]{2}{*}{ Region } & \multicolumn{3}{|c|}{$\mathrm{HC}$} & \multicolumn{3}{|c|}{ CBP } & \multirow[b]{2}{*}{ Type } \\
\hline & $\boldsymbol{k}_{i}$ & Module & $\left\langle k_{i}\right\rangle_{\text {Module }}$ & $\boldsymbol{k}_{\boldsymbol{i}}$ & Module & $\left\langle k_{i}\right\rangle_{\text {Module }}$ & \\
\hline ITG.L & 6.50 & Parieto-temporal & 2.33 & 4.25 & Visual & 3.20 & * \\
\hline ITG.R & 7.75 & Parieto-temporal & 2.33 & 6.00 & Visual & 3.20 & $*$ \\
\hline OrbSupMed.R & 4.67 & Fronto-medial & 3.21 & 3.92 & Orbito-frontal & 2.40 & $*$ \\
\hline MTG.R & 7.17 & Parieto-temporal & 2.33 & 7.42 & Fronto-parietal & 3.14 & $*$ \\
\hline OrbSup.L & 3.92 & Fronto-medial & 3.21 & 4.33 & Orbito-frontal & 2.40 & $*$ \\
\hline CAU.R & 1.75 & Subcortical & 2.40 & 2.42 & Subcortical & 2.58 & $\dagger$ \\
\hline ACG.L & 3.92 & Fronto-medial & 3.21 & 5.33 & Fronto-medial & 3.05 & $\dagger$ \\
\hline SFGmed.R & 5.42 & Fronto-medial & 3.21 & 5.17 & Fronto-medial & 3.05 & $\bullet$ \\
\hline
\end{tabular}

Different symbols indicate: ${ }^{*}$, global changes produced by a switch in module membership; $t$, local changes due to increment in number of connections of the node; -, changes due to reorganization of links from inter-module links to intra-module links. The node's degree and the mean module degree are also listed.

state network and pain processing. To our knowledge, this is the first report analyzing in detail the structure of these functional brain networks in CBP patients. Indeed, we found that functional brain networks in both healthy and CBP patients were well described by six different communities of modules: Visual (com1), fronto-medial (com2), parieto-temporal (com3), fronto-parietal (com4), subcortical (com5), and sensorimotor (com6). Moreover, it was observed that the modular organization of the functional brain networks in HC was in line with previous studies (Meunier et al., 2009a,b), describing a posterior community (similar to our visual community), a central community (including the sensorimotor and the subcortical communities), and an additional community involving frontal and cingulate cortices as well as the temporal lobes (similar to our frontomedial and parieto-temporal communities). As a further validation of our approach, we demonstrated that our visual, fronto-medial, parieto-temporal, and fronto-parietal communities were quite similar to the visual, default mode, executive control, and auditory resting state networks (Beckmann et al., 2005), respectively.

Differences between healthy subjects and CBP patients were mainly characterized by a reorganization of fronto-medial, parietotemporal, fronto-parietal, and sensorimotor communities involving frontal and temporal structures, as well as sensorimotor cortex, basal ganglia, insula, and anterior cingulate cortex (ACC). Basically, this reorganization implied a change in the module membership of the orbito-frontal cortex, the insula, and the posterior portion of the cingulate gyrus, which are involved in affective and cognitive processing, as well as in somatosensory integration. In addition, we found that caudate nucleus and ACC displayed a high inter-modular connectivity in CBP patients as compared with HC. The ACC has been identified as a key brain region in pain processing, responsible for the integration of sensory, attentional, emotional, and motivational aspects of pain; whereas the caudate nucleus participates in pain processing in sync with the ACC and several regions of the prefrontal cortex (Oshiro et al., 2009). Thus, it appears that chronic pain might be associated with changes in the modular organization and with an increased functional correlation of brain regions involved in processing and integration of sensory, affective, and cognitive components of pain. These findings are consistent with previous reports, showing that enduring pain might disrupt the functional connectivity of cortical regions of the DMN with the insula and the middle frontal gyrus (Baliki et al., 2008; Tagliazucchi et al., 2010), key regions which appear to be implicated in internally generated thought processes (Raichle 

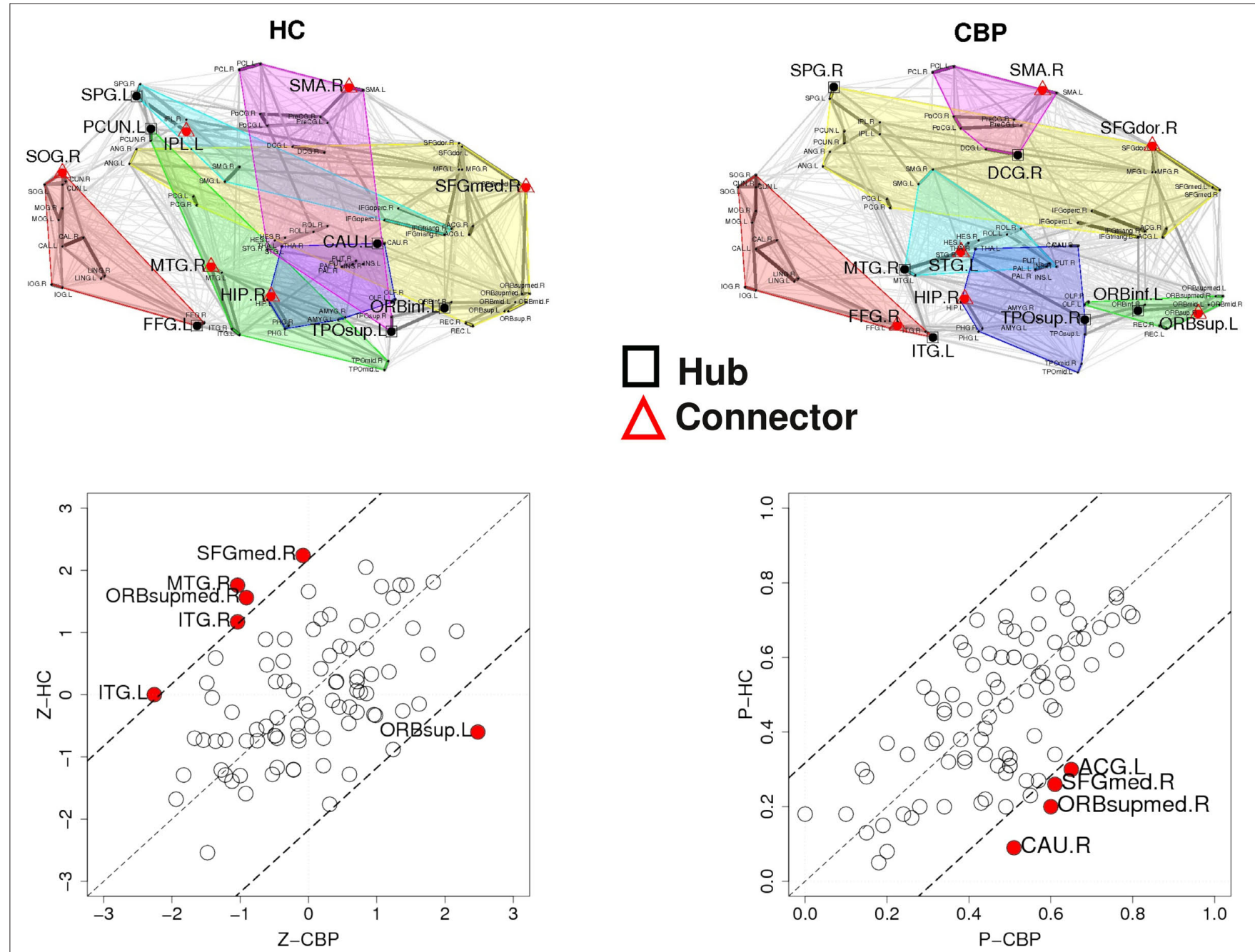

FIGURE 6 |Top panels: Hubs (nodes with highest intra-modular degree) and connectors (highest participation coefficient) in each module for both group networks. Bottom panels: Left: $z_{i,(\mathrm{HC})}$ versus $z_{i,(\mathrm{CBP})}$, right $P_{i, \mathrm{HC})}$ versus $P_{i,(\mathrm{CBP})}$. The regions which display a role's change larger than two standard deviations (dashed lines) are labeled and plotted using filled circles.

et al., 2001) and to play an important role in the evaluation of pain intensity and other somatic signals (Critchley et al., 2004). Moreover, it has been reported that irrespective of the location, nature or course of the different pain syndromes, chronic pain patients exhibit significant reductions of gray matter in cingulate cortex, orbito-frontal cortex, and insula (May, 2008). Accordingly, it seems reasonable that persistent, emotionally laden, self-reflective thoughts as it occurs in chronic pain states might have led to disrupt the modular organization of insula and orbito-frontal cortex and to increase the number of connections of ACC with the rest of the brain.

Our results show an organization comprising the same number of modules, and when changes were detected, they were well explained by straightforward pairwise changes in the linear correlation between a few nodes. Although caution should be always exercised, the fact that pairwise correlations were sufficient to explain global changes, suggests that correlations between a small number of key regions have a chance to be informative about the functional brain connectivity. Another implication of the present results relates to the physical substrate sustaining these dynamical changes, in particular since it has been previously described that chronic pain may elicit relevant changes in white and gray matter (Geha et al., 2008; May, 2008). These findings are consistent with the alterations in correlations described here, but further studies are needed to establish the validity and extent of the functional and structural relation. If proven true it will reinforce the idea that the stressful experience of enduring pain over many years could by itself modify adaptive synaptic connections, reshaping the connectivity of the subnetworks studied in the present study.

The present study has several limitations. An important one is the underlying assumption that chronic pain patient's brain is altered by the disease. Although this is a reasonable hypothesis it is yet far from proven and, in addition, even if there are alterations, still is unknown how large could be the individual variability. In other words, it is not yet known up to which point the potential changes in cortical dynamics in each individual are consistent across the patient's group, such that it can be detected by the kind of group average 
analyses performed here. Another important limitation is the common assumption considering the ninety anatomically defined brain areas as representing the centerpieces of a large-scale brain network. This is a good simplifying approach, that facilitates and compresses information, but it could be limiting our ability to detect differences between groups. One of the reasons is that regions selected by this method, despite covering very different sizes of cortical territories are all being equalized when considered as a single node in a network. Finally, another factor to consider is that the components obtained from an anatomical parcellation method do not necessarily mirror the dynamical components of brain activity as is shown in Figures A1 and $\mathbf{A} 2$ of Appendix where communities found in this work were compared with networks obtained from principal component analyses (as in Beckmann et al., 2005).

In summary, the study of brain correlation networks revealed an organization of up to six communities with similar modularity in both healthy and chronic pain groups. These differences appeared in the membership of key communities of frontal and temporal regions and confirmed by a surprisingly naive analysis based on pairwise correlations of the strongest and weakest correlated regions in HC. Besides confirming the brain effects of long enduring pain, our results provide a framework for studying the effects of other chronic conditions on functional brain networks.

\section{ACKNOWLEDGMENTS}

The authors thank the patients and the healthy volunteers for their participations in the study. Work supported by NIH NINDS of USA, grant No. NS58661, by CONICET (grant No. PIP0802/10 and PIP0087), MinCyt (Grant No. 863) and University of Buenos Aires (grant No. UBACyT X-404), Argentina and by Spanish Ministry of Science and European Funds FEDER (grant No. SEJ2007-62312). Funding for data collection was provided by grant No. NS35115 (awarded to A.V. Apkarian) by NIH NINDS.

\section{REFERENCES}

Achard, S., and Bullmore, E. T. (2007). Efficiency and cost of economical brain functional networks. PLoS Comput. Biol. e17. doi: 10.1371/ journal.pcbi.0030017.

Ahnert, S. E., Garlaschelli, D., Fink, T. M. A., and Caldarelli, G. (2007). Ensemble approach to the analysis of weighted networks. Phys. Rev. E. 76, 016101-016105.

Apkarian, A. V., Sosa, Y., Krauss, B. R., Thomas, P. S., Fredrickson, B. E., Levy, R. E., Harden, R., and Chialvo, D. R. (2004). Chronic pain patients are impaired on an emotional decisionmaking task. Pain 108, 129-136.

Baliki, M. N., Chialvo, D. R., Geha, P. Y., Levy, R. M., Harden, R. N., Parrish, T. B., and Apkarian,A.V. (2006).Chronic pain and the emotional brain: specific brain activity associated with spontaneous fluctuations of intensity of chronic back pain. J. Neurosci. 26, 12165-12173.

Baliki, M. N., Geha, P. Y., Apkarian, A. V., and Chialvo, D. R. (2008). Beyond feeling: chronic pain hurts the brain, disrupting the default-mode network dynamics. J. Neurosci. 28, 1398-1403.

Beckmann, C. F., DeLuca, M., Devlin, J. T., and Smith, S.M. (2005). Investigations into resting-state connectivity using independent component analysis. Philos. Trans. R. Soc. Lond. B. Biol. Sci. 360, 1001-1013.

Beckmann, C. F., and Smith, S. M. (2004). Probabilistic independent component analysis for functional magnetic resonance imaging. IEEE Trans. Med. Imag. 23, 137-152.

Boettcher, S., and Percus, A. G. (2001a). Optimization with extremal dynamics. Phys. Rev. Lett. 86, 5211-5214.
Boettcher, S., and Percus, A. G. (2001b). Extremal optimization for graph partitioning. Phys. Rev. E. 64, 026114-026126.

Broyd, S. J., Demanuele, C., Debener, S., Helps, S. K., James, C. J., and SonugaBarke, E. J. S. (2009). Default-mode brain dysfunction in menthal disorders: a systematic review. Neurosci. Biobehav. Rev. 33, 279-296.

Bullmore, E. T., and Sporns, O. (2009). Complex brain networks: graphtheoretical analysis of structural and functional systems. Nat. Rev. Neurosci. 10, 186-198.

Clauset, A., Newman, M. E. J., and Moore, C. (2004). Finding community structure in very large networks. Phys. Rev. E. 70, 066111-066116.

Cordes, D., Haughton, V. M., Arfanakis, K., Carew, J. D., Turski, P. A., Moritz, C. H., Quigley, M. A., and Meyerand, M. E. (2001). Frequencies contributing to functional connectivity in the cerebral cortex in "resting-state" data. Am. J. Neuroradiol. 22, 1326-1333.

Cordes, D., Haughton, V. M., Arfanakis, K., Wendt, G. J., Turski, P. A., Moritz, C. H., Quigley, M. A., and Meyerand, M. E. (2000). Mapping functional related regions of brain with functional connectivity MR imaging. Am. J. Neuroradiol. 21, 1636-1644.

Critchley, H. D., Wiens, S., Rotshtein, P., Ohman, A., and Dolan, R. J. (2004). Neural systems supporting interoceptive awareness. Nat. Neurosci. 7, 189-195.

Damoiseaux, J. S., Rombouts, S. A. R. B., Barkhof, F., Scheltens, P., Stam, C. J., Smith, S. M., and Beckmann, C. F. (2006). Consistent resting-state networks across healthy subjects. Proc. Natl. Acad. Sci. U.S.A. 103, 13848-13853.
Duch, J., and Arenas, A. (2005). Community detection in complex networks using extremal optimization. Phys. Rev. E. 72, 027104-027107.

Eguiluz, V. M., Chialvo, D. R., Cecchi, G., Baliki, M., and Apkarian, A. V. (2005). Scale-free brain functional networks. Phys. Rev. Lett. 94, 018102-018105.

Fox, M. D., Snyder, A. Z., Vincent, J. L., Corbetta, M., van Essen, D. C., and Raichle, M. E. (2005). From the cover: the human brain is intrinsically organized into dynamic, anticorrelated functional networks. Proc. Natl. Acad. Sci. U.S.A. 102, 9673-9678.

Fox, M. D., and Raichle, M. E. (2007). Spontaneous fluctuations in brain activity observed with functional magnetic resonance imaging. Nat. Rev. Neurosci. 8, 700-711.

Garrity, A. G., Pearlson, G. D., McKiernan, K., Lloyd, D., Kiehl, K. A., and Calhoun, V.D. (2007). Aberrant "default mode" functional connectivity in schizophrenia. Am. J. Psychiatry 164, 450-457.

Geha, P. Y., Baliki, M. N., Harden, R. N., Bauer, W. R., Parrish, T. B., and Apkarian, A. V. (2008). The brain in chronic CRPS pain: abnormal graywhite matter interactions in emotional and autonomic regions. Neuron 60, 570-581.

Guimera, R., and Amaral, L. A. N. (2005). Functional cartography of complex metabolic networks. Nature 433, 895-900.

He, Y., Chen, Z., and Evans, A. (2008) Structural insights into aberrant topological patterns of large-scale cortical networks in Alzheimer's disease. J. Neurosci. 28, 4756-4766.

Jezzard, P., Mathews, P., and Smith, S. M. (2001). Functional MRI: An Introduction to Methods. Oxford: Oxford University Press.
Laufs, H., Hamandi, K., Salek-Haddadi, A., Kleinschmidt, A., Duncan, J. S., and Lemieux, L. (2007). Temporal lobe interictal epileptic discharges affect cerebral activity in default mode brain regions. Hum. Brain. Mapp. 28, 1023-1032.

Lui, S.,Ouyang, L., Chen, Q., Huang, X., Tang, H., Chen, H., Zhou, D., Kemp, G. J., and Gong, Q. (2008). Differential interictal activity of the precuneus/posterior cingulate cortex revealed by resting state fMRI at $3 \mathrm{~T}$ in generalized versus partial seizure. J. Magn. Res. Imag. 27, 1214-1220.

May, A. (2008). Chronic pain may change the structure of the brain. Pain 137, 7-15.

Meunier, D., Achard, S., Morcom, A., and Bullmore, E. T. (2009a). Age-related changes in modular organization of human brain functional networks. Neuroimage 44, 715-723.

Meunier, D., Lambiotte, R., Fornito, A., Ersche, K., and Bullmore, E. T. (2009b). Hierarchical modularity in human brain functional networks. Front. Neuroinform. 3:37.doi: 10.3389/ neuro.11.037.2009.

Newman, M. E. J. (2004). Analysis of weighted networks. Phys. Rev. E. 70, 056131-056139.

Newman, M. E. J., and Girvan, M. (2004). Finding and evaluating community structure in networks. Phys. Rev. E. 69, 026113-026127.

Oshiro, Y., Quevedo, A. S., McHaffie, J. G., Kraft, R. A., and Coghill, R. C. (2009). Brain mechanisms supporting discrimination of sensory features of pain: a new model. J. Neurosci. 29, 14924-14931.

PICA. http://www.fmrib.ox.ac.uk/ analysis/research/melodic. 
Rand, W. M. (1971). Objective criteria for the evaluation of clustering methods. J. Amer. Stat. Assoc. 66, 846-850.

Raichle, M. E., MacLeod, A. M., Snyder, A. Z., Powers, W. J., Gusnard, D. A., and Shulman, G.L. (2001). A default mode of brain function. Proc. Natl. Acad. Sci. U.S.A. 98, 676-682.

Salvador, R., Suckling, J., Coleman, M. R., Pickard,J.D., Menon, D., and Bullmore, E. T. (2005). Neurophysiological architecture of functional magnetic resonance images of human brain. Cereb. Cortex 15, 1332-1342.

Smith, S. M., Fox, P. T., Miller, K. L., Glahn, D. C., Fox, P. M., Mackay, C. E., Filippini, N., Watkins, K. E., Toro, R., Laird, A. R., and Beckmann, C.F. (2009). Close correspondence of major functional networks in rest and under task. Proc. Natl. Acad. Sci. U.S.A. 106, 13040-13045.

Soranzo, N., Bianconi, G., and Altafini, C. (2007). Comparing association network algorithms for reverse engineering of large-scale gene regulatory networks: synthetic versus real data. Bioinformatics 23, 1640-1647.

Sporns, O., Chialvo, D. R., Kaiser, M., and Hilgetag, C. C. (2004). Organization, development and function of complex brain networks. Trends Cogn. Sci. 8, 418-425.

Stanberry, L., Murua, A., and Cordes, D. (2008). Functional connectivity mapping using the ferromagnetic Potts spin model. Hum. Brain Mapp. 29, 422-440.

Tagliazucchi, E., Balenzuela, P., Fraiman, D., Chialvo, D. R. (2010). Brain resting state is disrupted in chronic back pain patients. Neurosci. Lett. 485, 26-31.

Tracey, I., and Mantyh, P. W. (2007). The cerebral signature for pain perception and its modulation. Neuron 55, 377-391.

Tzourio-Mazoyer, N., Landeau, B., Papathanassiou, D., Crivello, F., Etard,
O., Delcroix, N., Mazoyer, B., and Joliot, M. (2002). Automated anatomical labeling of activations in SPM using a macroscopic anatomical parcellation of the MNI MRI single-subject brain. Neuroimage 15, 273-289.

van den Heuven, M. P., Stam, C. J., Boersma, M., and Hullshof Pol, H. E. (2008). Small-world and scalefree organization of voxel-based resting-state functional connectivity in the human brain. Neuroimage 43, 528-539.

Witting, N., Kupers, R. C., Svensson, P., Jensen, T. S. (2006). A PET activation study of brush-evoked allodynia in patients with nerve injury pain. Pain 120, 145-154.

Zaki, J., Ochsner, K. N., Hanelin, J., Wager, T. D., and Mackey, S. C. (2007). Different circuits for different pain: Patterns of functional connectivity reveal distinct networks for processing pain in self and others. Soc. Neurosci. 2, 276-291.
Conflict of Interest Statement: The authors declare that the research was conducted in the absence of any commercial or financial relationships that could be construed as a potential conflict of interest.

Received: 26 February 2010; accepted: 18 October 2010; published online: 17 November 2010.

Citation: Balenzuela P, Chernomoretz A, Fraiman D, Cifre I, Sitges C, Montoya P and Chialvo DR (2010) Modular organization of brain resting state networks in chronic back pain patients. Front. Neuroinform. 4:116. doi: 10.3389/fninf.2010.00116 Copyright (c) 2010 Balenzuela, Chernomoretz, Fraiman, Cifre, Sitges, Montoya and Chialvo. This is an openaccess article subject to an exclusive license agreement between the authors and the Frontiers Research Foundation, which permits unrestricted use, distribution, and reproduction in any medium, provided the original authors and source are credited. 


\section{APPENDIX}

Table A1 | Cortical and subcortical regions in each hemisphere as defined in Tzourio-Mazoyer et al. (2002), abbreviations as in Salvador et al. (2005).

\section{Region}

Precentral gyrus

Superior frontal gyrus, dorsolateral

Superior frontal gyrus, orbital part

Middle frontal gyrus

Middle frontal gyrus, orbital part

Middle frontal gyrus, opercular part

Inferior frontal gyrus, triangular part

Inferior frontal gyrus, orbital part

Rolandic operculum

Supplementary motor area

Olfactory cortex

Superior frontal gyrus, medial

Superior frontal gyrus, medial orbital

Gyrus rectus

Insula

Anterior cingulate and paracingulate gyri

Median cingulate and paracingulate gyri

Posterior cingulate gyrus

Hippocampus

Parahippocampal gyrus

Amygdala

Calcarine fissure and surrounding cortex

Cuneus

Lingual gyrus

Superior occipital gyrus

Middle occipital gyrus

Inferior occipital gyrus

Fusiform gyrus

Postcentral gyrus

Superior parietal gyrus

Inferior parietal, but supramarginal and angular gyri

Supramarginal gyrus

Angular gyrus

Precuneus

Paracentral lobule

Caudate nucleus

Lenticular nucleus, putamen

Lenticular nucleus, pallidum

Thalamus

Heschl gyrus

Superior temporal gyrus

Temporal pole: superior temporal gyrus

Middle temporal gyrus

Temporal pole: middle temporal gyrus

Inferior temporal gyrus

\section{Abbreviation}

PreCG

SFGdor

ORBSUP

MFG

ORBmid

IFGoperc

IFGtriang

ORBinf

ROL

SMA

OLF

SFGmed

ORBsupmed

REC

INS

ACG

DCG

PCG

HIP

PHG

AMYG

CAL

CUN

LING

SOG

MOG

IOG

FFG

PoCG

SPG

IPL

SMG

ANG

PCUN

PCL

CAU

PUT

PAL

THAL

HES

STG

TPOsup

MTG

TPOmid

ITG 
Table A2 | List of regions composing each of the six communities found for the brain networks in each group.

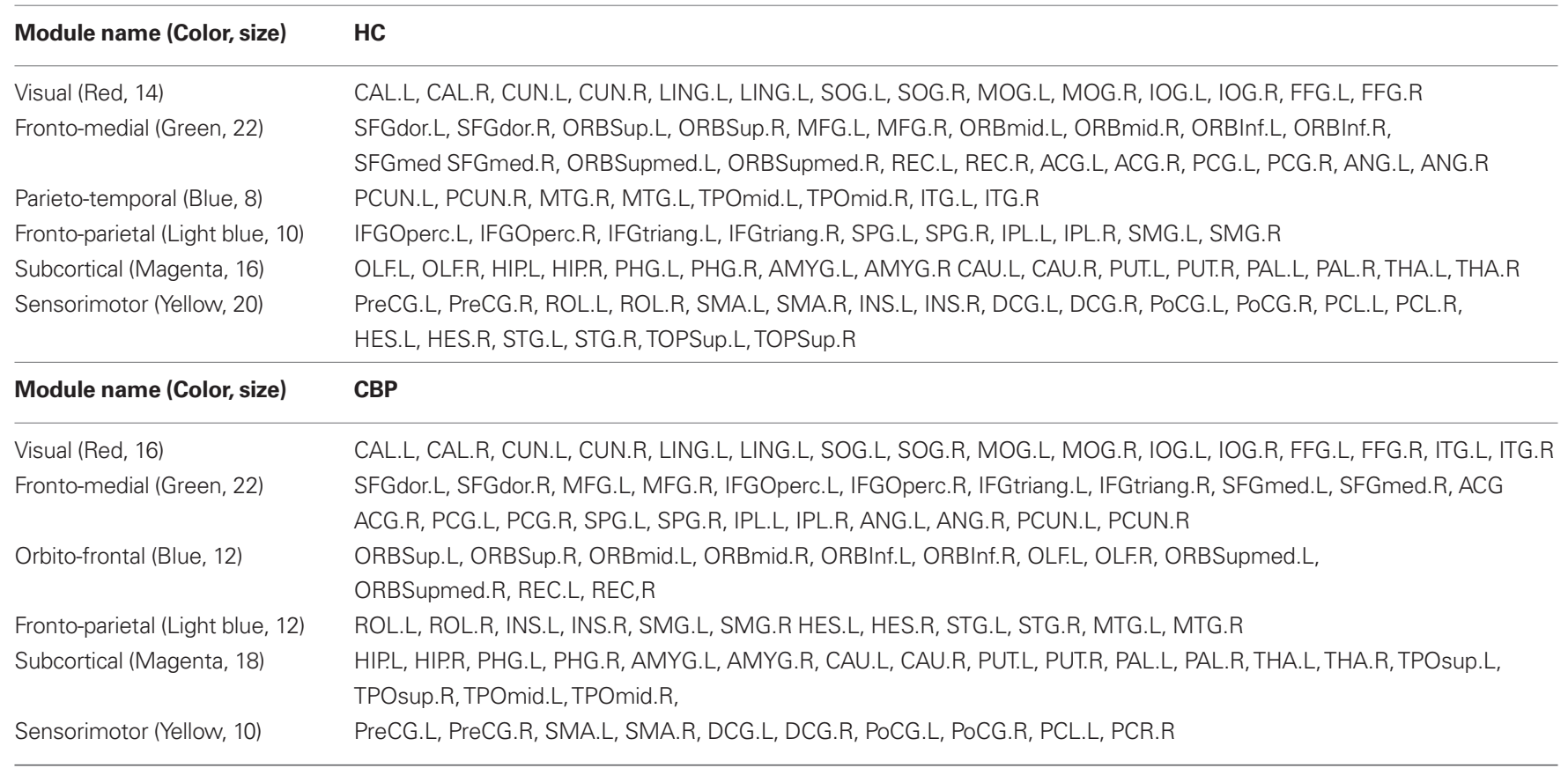

The color code used to label each community in the figures is indicated as well 


\section{Healthy Controls}

VISUAL

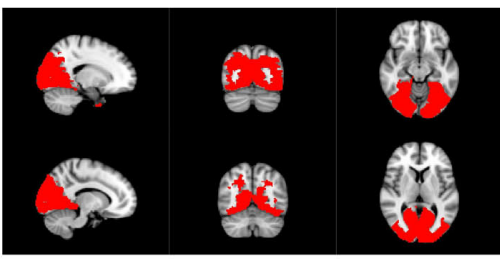

FRONTO-

MEDIAL
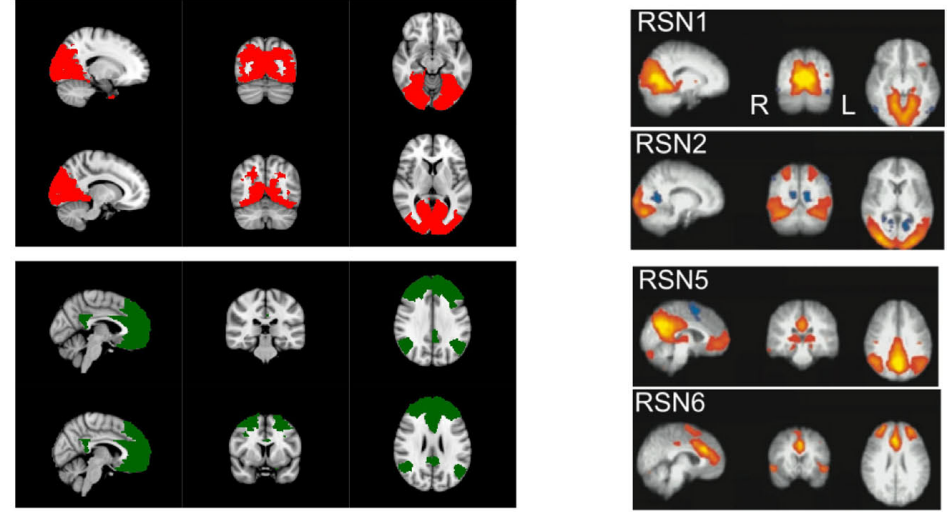

PARIETO-

TEMPORAL
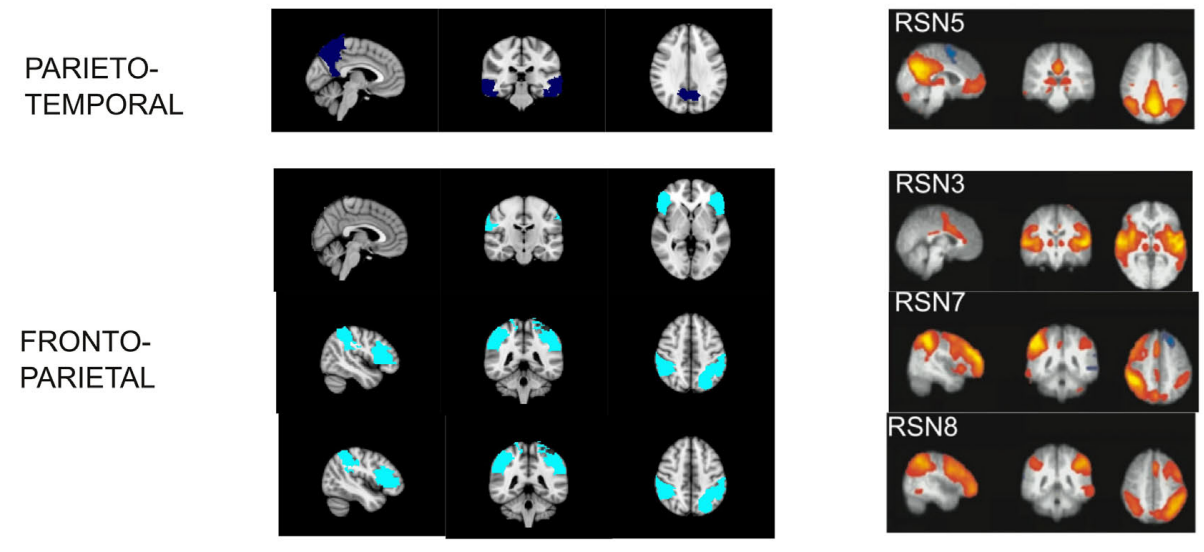

SUBCORTICAL

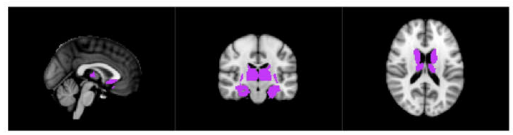

SENSORIMOTOR
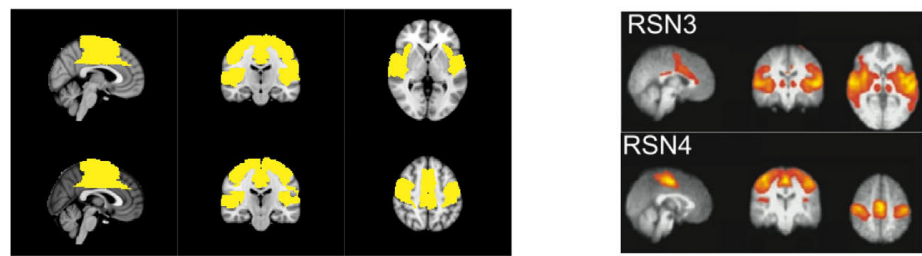

FIGURE A1 | Communities found in normal group (HC) compared with resting state networks obtained from probabilistic component analysis in Beckmann et al. (2005). These regions, associated with specific cognitive functions, are labeled as follows: RSN1 (medial areas of the visual cortex), RSN2 (lateral areas of the visual cortex), RSN3 (auditory), RSN4 (sensory motor), RSN5 (default mode network), RSN6 (executive control), RSN7 (right dorsal visual stream), RSN8 (left dorsal visual stream). Coordinates used are: RSN1, $x=17$ $y=-73 z=-12$; RSN2, $x=-13 y=-61 z=6$; RSN3: $x=3, y=-17, z=1.5$;

RSN4, $x=1, y=-21, z=51$; RSN5, $x=-4, y=-29, z=33 ;$ RSN6, $x=5, y=6$, $z=27 ; \operatorname{RSN7}, x=45, y=-42, z=47 ; \operatorname{RSN} 8, x=-45, y=-42, z=47$. 


\section{CBP Patients}

VISUAL

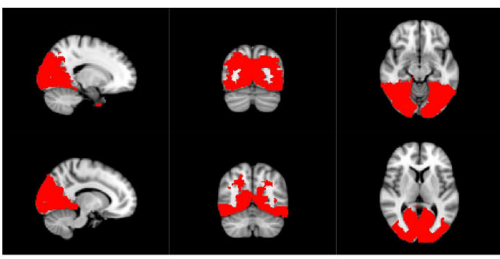

FRONTOMEDIAL
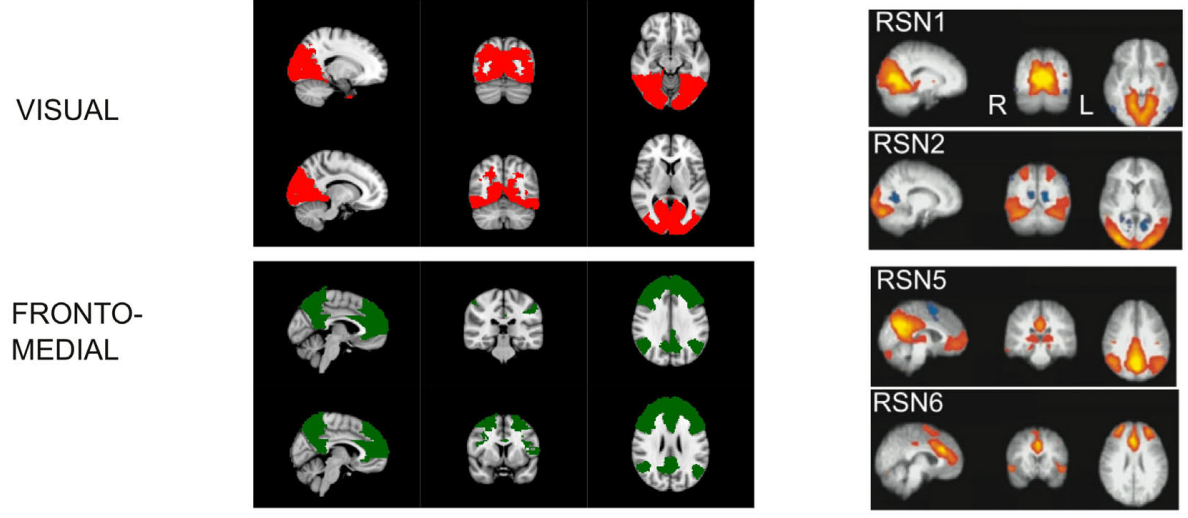

ORBITO-

FRONTAL
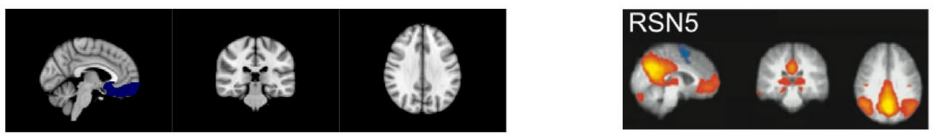

FRONTO-
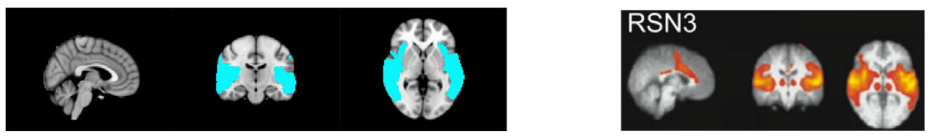

PARIETAL
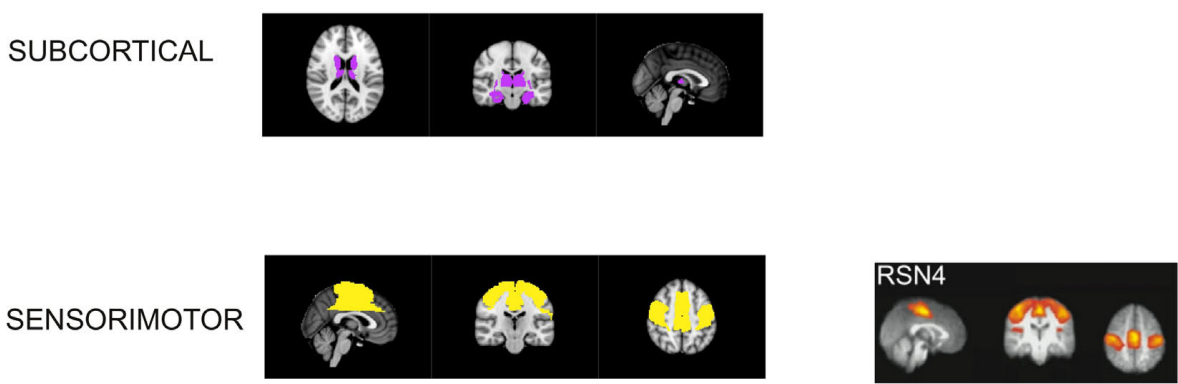

FIGURE A2 | Communities found in chronic back pain group (CBP) compared with resting state networks obtained from probabilistic component analysis in Beckmann et al. (2005). These regions, associated with specific cognitive functions, are labeled as follows: RSN1 (medial areas of the visual cortex), RSN2 (lateral areas of the visual cortex), RSN3 (auditory), RSN4 (sensory motor), RSN5 (default mode network), RSN6 (executive control), RSN7 (right dorsal visual stream), RSN8 (left dorsal visual stream). Coordinates used are the same as in Figure S1.

\section{REFERENCES}

Baliki,M.N.,Chialvo,D.R., Geha,P.Y.,Levy, R. M., Harden, R. N., Parrish, T. B., and Apkarian, A. V. (2006). Chronic pain and the emotional brain: specific brain activity associated with spontaneous fluctuations of intensity of chronic back pain. J. Neurosci. 26, 12165-12173.
Baliki, M. N., Geha, P. Y., Apkarian, A. V., and Chialvo, D. R. (2008). Beyond feeling: chronic pain hurts the brain, disrupting the default-mode network dynamics. J. Neurosci. 28, 1398-1403.

Fox, M. D., and Raichle, M. E. (2007). Spontaneous fluctuations in brain activity observed with functional magnetic resonance imaging. Nat. Rev Neurosci. 8, 700-711.

Salvador, R., Suckling, J., Coleman, M. R., Pickard,J.D., Menon, D., and Bullmore, E. T. (2005). Neurophysiological architecture of functional magnetic resonance images of human brain. Cereb. Cortex 15, 1332-1342.
Tzourio-Mazoyer, N., Landeau, B., Papathanassiou, D., Crivello, F., Etard, O., Delcroix, N., Mazoyer, B., and Joliot, M. (2002). Automated anatomical labeling of activations in SPM using a macroscopic anatomical parcellation of the MNI MRI single-subject brain. Neuroimage 15, 273-289. 